\title{
The expected isotropic distribution curves in galactic alignment (Research Note)
}

\author{
G. X. Wu \\ Purple Mountain Observatory, Chinese Academy of Sciences, Nanjing 210008, PR China \\ e-mail: gxwu@pmo.ac.cn \\ Received 7 December 2005 / Accepted 4 February 2006

\section{ABSTRACT} \\ Galactic alignment may be a probe of galaxy formation. In such studies, comparisons between the observational distribution of the \\ spin vectors of galaxies and the assumed isotropic distribution are usually performed to try to decide whether alignment exists or not. \\ It has been pointed out that the isotropic distribution that is usually adopted suffers from severe selection effects for the location of \\ cluster and inclination of galaxies. We repeated this simulation for the expected isotropic distribution, and these selection effects were \\ confirmed. Moreover, our results show that the selection effect for the location of cluster can be sidestepped by using the appropriate \\ reference frame, if there is no selection for inclination. This result is especially useful in studies of galactic alignment using samples \\ measured by fitting a certain isophotal level.
}

Key words. galaxies: cluster: general - galaxies: statistics

\section{Introduction}

Rotation about a fixed axis is one of the fundamental properties of spiral galaxies. Some arguments exist to show that the tidal effects in the epoch of post-formation would not significantly perturb these dynamical axes in almost all environments (Thompson 1976; Farouki \& Shapiro 1981; Djorgovski 1987). Accordingly, the directions of the spin vectors of galaxies may be a kind of dynamical fossil, representing to some extent the initial conditions for the formation of galaxies and galaxy clusters. Investigating the alignment of the spin vectors of galaxies thus may be a powerful cosmological test that can be used to confront the theories of structure formation with observations.

Theoretically, various theories of structure formation predict a diverse distribution of the spin vectors of galaxies, and there are mainly three competing theories for this. First, in the "topdown" scenario (Zeldovich 1978; Doroshkevich \& Shandarin 1978), the spin vectors of galaxies preferentially lie within the cluster plane. Second, the "bottom-up" scenario (Peebles 1969, 1974) predicts a random distribution. Third, in the primordial vorticity theory (Ozernoy 1978), the spin vectors of galaxies tend to be perpendicular to the cluster plane. It should be noted that structure formation is a complex process, since the phenomena involved in the other theories may all play a role.

On the observational side, the search for galactic alignment has a long history (Djorgovski 1987). In the pioneering days, the position angle (PA) and axial ratio distribution were inspected independently. The alignment effect may be diluted by the projection effect in this method. Later, Jaaniste \& Saar (1978) and Flin \& Godlowski (1986) proposed a PA-inclination method, in which the position angle and axial ratio are considered simultaneously. In this method, the galactic alignment is examined by the comparison between observational distribution of the spin vectors of galaxies and the expected isotropic distribution.

The expected isotropic distribution have been carefully studied by Aryal \& Saurer (2000), who show that selection effects for the cluster location (limited sky coverage) and inclination (lack of PAs for face-on galaxies) may substantially alter the shape of the expected isotropic distribution; the different sky regions under study correspond to the different isotropic distribution that are expected. They claim that this selection effect is unavoidable for the samples taken from a limited region of the sky, so that the simulated expected isotropic distribution should be used. In this paper, however, we argue that the selection effect concerning the cluster location can be sidestepped by using the appropriate reference frame. The method for calculating the spin vectors of galaxies is described in Sect. 2. We give the simulation for the expected isotropic distribution and suggest a way to sidestep this selection effect in Sect. 3. Finally, some discussions are given is Sect. 4

\section{Calculation of the spin vectors}

The spin vectors of galaxies were calculated using the PA-inclination method in the most recently published papers. In this method, the two-dimensional parameters, such as PA and axial ratio, are used to derive the directions of the spin vectors of galaxies in a specific reference frame. A Local Supergalactic coordinate system is commonly adopted, in which the spin vector of a galaxy is expressed by polar angle $\theta$ and azimuthal angle $\phi$. The formulae to calculate $\theta$ and $\phi$ are as follows (Flin \& Godlowski 1986):

$$
\begin{aligned}
\sin \theta= & -\cos i \sin B \pm \sin i \sin P \cos B \\
\sin \phi= & (\cos \theta)^{-1}[-\cos i \cos B \sin L \\
& +\sin i(\mp \sin P \sin B \sin L \mp \cos P \cos L)]
\end{aligned}
$$

where $L, B$, and $P$ are longitude, latitude, and PA in supergalactic system, respectively. Inclination angle $i$ is calculated by the formula $\cos ^{2} i=\left[(b / a)^{2}-0.2^{2}\right] /\left(1-0.2^{2}\right)$, with $b / a$ as the axial ratio (Holmberg 1946).

It should be noted that these formulae give two pairs of $\theta$ and $\phi$ for a given $i$, corresponding to four solutions for the spin vectors of each galaxy. As usual, these four solutions are all counted independently. Instead of producing artifical anisotropy, this procedure tends to increase isotropy (Flin \& Godlowski 1989), so any positive results for galactic alignment could not be affected by this four-solution ambiguity. 
It is obvious that the solutions for $\theta$ and $\phi$ strongly depend on the positions of galaxies. Flin \& Godlowski (1986) propose two analytical methods to reduce this influence. We take the absolute values of $\theta$, and consider $\phi$ in the interval of $-90^{\circ}$ to $90^{\circ}$ (Kashikawa \& Okamura 1992). However, as shown in Aryal \& Saurer (2000), this method can be used to reduce the influence on the expected isotropic distribution only when the galaxies are distributed over the whole celestial sphere. For a limited sky region, even if there is no selection for galactic inclination, the usually adopted expected isotropic distribution could be affected.

\section{Simulation of the expected isotropic distribution}

The widely used process for studying of galactic alignment is as follows. First, the polar angle $\theta$ and azimuthal angle $\phi$ of the spin vectors of galaxies are calculated using formulae (1) and (2), then the calculated values are binned to form the observational distribution. Based on the comparison between observational distribution and the assumed expected isotropic distribution, we then try to decide upon isotropy or anisotropy; any deviation from the expected isotropic distribution is regarded as evidence for anisotropy. The usually adopted isotropic distribution uses one cosine curve and one straight line for $\theta$ and $\phi$, respectively.

Aryal \& Saurer (2000) propose another choice for the expected isotropic distribution. Considering the fact that $\theta$ and $\phi$ calculated using formulae (1) and (2) strongly depend on the positions of galaxies, for an assumed cluster with member galaxies whose spin vectors are isotropic in space, they claim that the distribution of $\theta$ and $\phi$ for galaxies in this cluster would dependent on the location of this cluster in the sky. On the other hand, the distribution of $\theta$ and $\phi$ of this assumed cluster are considered as the expected isotropic distribution for this region in the sky. According to this point,different regions in the sky have different expected isotropic distributions, so it is necessary to use simulated expected isotropic curves to study a limited region in the sky. This is the so-called selection effect for cluster location.

We follow the method of Aryal \& Saurer (2000) for simulating the expected isotropic curves. If the spin vectors of galaxies are assumed to be isotropic in space, then because of projection effect, the probability functions of $i, B, L$, and $P$ would be $\propto \sin i$, $\propto \cos B, \propto 1$, and $\propto 1$, respectively. We apply formulae (1) and (2) to calculate $\theta$ and $\phi$ for $2 \times 10^{5}$ virtual galaxies to obtain the expected isotropic curves for a specific region in the sky.

As a first step, we repeat the simulation of Aryal \& Saurer (2000) to obtain the identical results. Because the PA and the axial ratio in modern catalogues is almost always measured by fitting a certain isophotal level, where there is no selection for inclination, we pay attention to the selection effect for cluster location. Figure 2a of Aryal \& Saurer (2000) obviously shows that the location of the region under consideration strongly changes the shape of the expected isotropic curves of $\phi$. As an example, Fig. 1 shows the expected isotropic curves of $\theta$ and $\phi$ for a region of $30^{\circ} \times 30^{\circ}$ located at $L=45^{\circ}, B=45^{\circ}$. As expected by the results of Aryal \& Saurer (2000), the distribution of $\theta$ remains a cosine, for there is no selection for inclination, but the distribution of $\phi$ obviously deviates from a straight line.

The PA-inclination method described in Flin \& Godlowski (1986) adopts the plane of the Local Supercluster as the reference plane. It is reasonable to think that the clusters outside the Local Supercluster, or even those within the Local Supercluster, have their own preferential planes that are not parallel to the plane of the Local Supercluster. To study the alignment effect
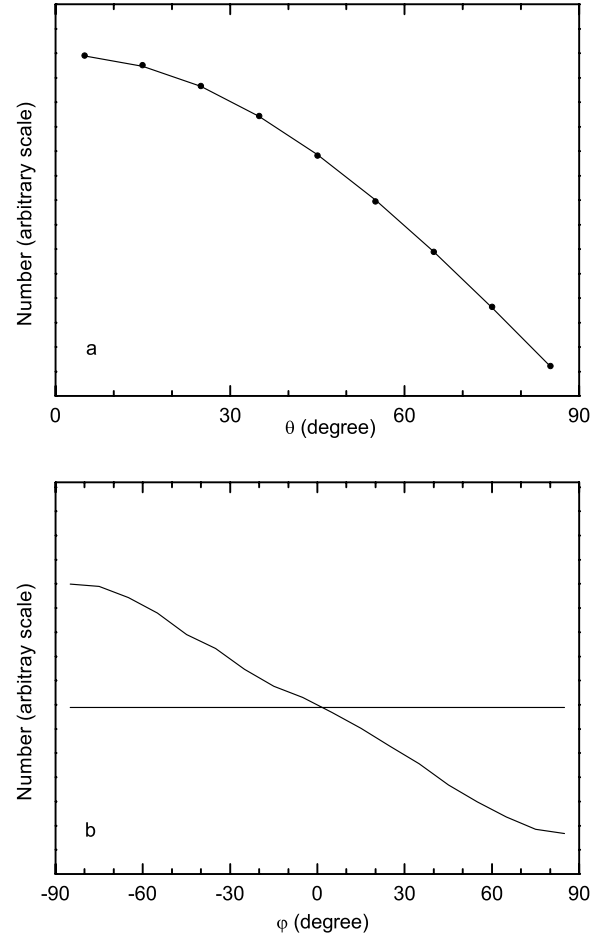

Fig. 1. The expected distribution of the polar angle $\theta$ (a) and azimuthal angle $\phi(\mathbf{b})$ for a region of $30^{\circ} \times 30^{\circ}$, located at $L=45^{\circ}, B=45^{\circ}$. The dots represent calculated values.

of those clusters with respect to their own plane may be more appropriate. The reference plane described in Flin \& Godlowski (1986) can be changed, and other corresponding changes should also be taken for some basic points. Using the cluster plane as the reference was first pointed out by Godlowski (1995), and has been used in studies of Hercules supercluster (Flin 1994), Coma/A1367, Perseus superclusters (Flin 2001, 1988), and for Tully group of galaxies (Godlowski \& Ostrowski 1999).

Keeping this in mind, we attempt to let the axis of $L=$ 0 point in the direction of the center of the region mentioned above. This is the same as to study a region of $30^{\circ} \times 30^{\circ}$ located at $L=0^{\circ}, B=45^{\circ}$. The expected distribution is shown in Fig. 2. Because the isotropic polar angle distribution is independent of $L$ and $B$ when there is no selection for $i$, the distribution of $\theta$ in Fig. 2a is a cosine curve, which is confirmed by the $\chi^{2}$-test. This gives $p=0.18$, which is higher than the commonly adopted significance level of 0.05. However, the distribution of $\phi$ in Fig. $2 b$ changes to a straight line, and the $\chi^{2}$-test gives $p=0.62$, significantly larger than 0.05 . In fact we also note that the sum of the two curves corresponding to $L=0^{\circ}-30^{\circ}$ and $L=330^{\circ}-360^{\circ}$ in Fig. 2a of Aryal \& Saurer (2000) leads to a straight line. Our other simulations for various $B$ show that the expected isotropic curves remain a cosine for $\theta$ and straight lines for $\phi$, provided the axes of $L=0$ point in the direction of the center of the region under study. This result demonstrates that by using the appropriate coordinate system, the selection effect for cluster location can be removed, and the simulation of the expected isotropic curves is not always necessary in these situations.

It is worthwhile pointing out that letting axis of $L=0$ point to the center of a cluster does not mean using the cluster plane as the reference. To do this, the pole to the cluster plane should be determined by another method, but this is beyond the scope of this paper. Letting the axis of $L=0$ point to the cluster center would remove the selection effect for cluster location. 

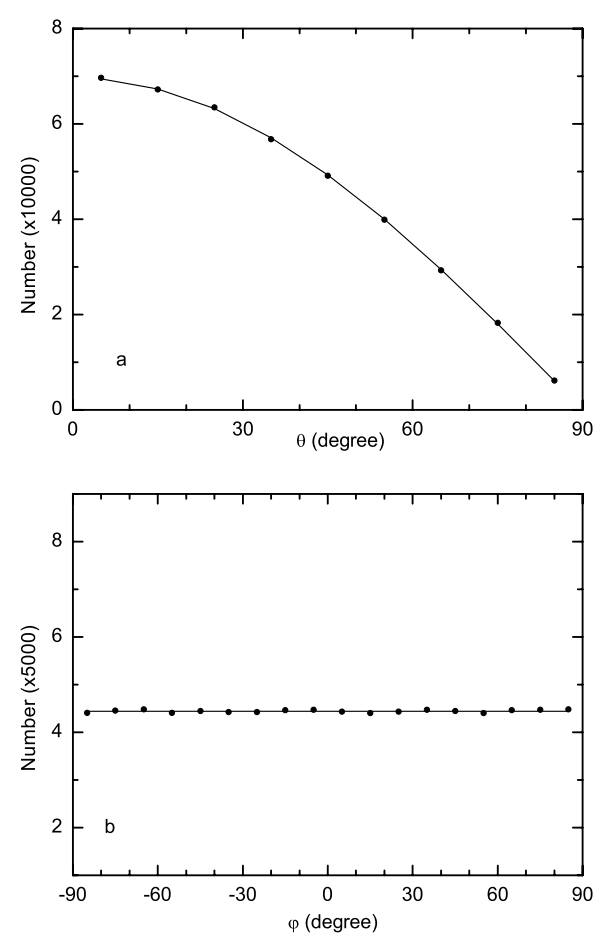

Fig. 2. The expected distribution of the polar angle $\theta$ (a) and azimuthal angle $\phi(\mathbf{b})$ for a region of $30^{\circ} \times 30^{\circ}$, located at $L=0^{\circ}, B=45^{\circ}$. The dots represent calculated values.

\section{Discussion and conclusion}

The choice of the expected isotropic distribution is important, because it relates directly to the results. Some studies have adopted a cosine curve and a straight line as the expected isotropic distribution for $\theta$ and $\phi$ (Flin \& Godlowski 1986; Kashikawa \& Okamura 1992; Godlowski 1993, 1994; Hu et al. 1995, 1998; Yuan et al. 1997; Wu et al. 1997). This choice stems from a simple consideration of the isotropic distribution of the polar and azimuthal angles of vectors within three-dimensional space, which have nothing to do with the specific arrangement of galaxy distribution. This choice may suffer from a selection effect due to limited sky coverage (Aryal \& Saurer 2000), but it cannot play a role in some studies of the Local Supercluster (e.g. Godlowski 1993, 1994), because there is no selection for $L$.

Other papers have used the simulated expected isotropic curves (Aryal \& Saurer 2004, 2005a,b,c). We note that the summation of the simulated distribution of $\phi$ for each region over the whole sky is a straight line, which is absolutely isotropic. It seems that the simulated distribution of $\phi$ for one region is a subsample of an isotropic distribution. On the other hand, when assuming a cluster filled with galaxies whose spin vectors are isotropic in space, it is hard to think that changes in the location of this assumed cluster would change the isotropic character of the distribution of $\phi$, for at this circumstance the distribution of $L, B$, and even PA would also be changed with the changes in the cluster location. While a straight line tends to test the isotropy, we suspect that the simulated distribution of $\phi$ tends to test the uniformity over the whole sky; it tests whether the observational distribution is a part of an isotropic distribution over the whole sky. However, the method described here would remove this paradox.

The simulated expected isotropic distribution may be affected by two aspects. One is the true shape of galaxies
(Godlowski \& Ostrowski 1999; Godlowski et al. 2005). The directions of the spin vectors depend on inclination of galaxies, which are calculated by the Holmberg formula with a given intrinsic flatness of galaxies (Holmberg 1946). However, this flatness depend on the galactic morphological type (e.g. Sandage et al. 1970), so it is hard to deal with this objectively in the simulation. Another problem is the position of galaxies. Once the spin vectors of galaxies depend on the position of galaxies, the assumption of a random distribution of the position of galaxies in a simulation may raise some systematic effect, because the distribution of galaxies in a real cluster is obviously not random. Considering that the reported galactic alignment effect is around a significance level of $2 \sigma$, it may be more objective to use a straight line to test whether the distribution of $\phi$ is isotropic or not.

In summary, it is shown that the expected isotropic curves remain a cosine curve and a straight line for polar angle $\theta$ and azimuthal angle $\phi$, if we let the axis of $L=0$ point to the direction of the center of the region under study; hence, even for samples with limited sky coverage, the selection effect for cluster location is removed. Some earlier studies taking the basic great "meridian" passing through the center of the investigated region would not suffer from this selection effect. This method is useful for samples of galaxies taken from catalogues in which PA and axial ratio are measured by fitting a given isophotal level. With this condition, we advocate using a reference frame with axis of $L=0$ pointing to the direction of the center of the investigated region to study galactic alignment effect.

Acknowledgements. We thank Prof. S. Okamura, F. X. Hu, B. Aryal, and W. Saurer for useful discussions during the preparation of a review paper. We thank the referee, Dr. Godlowski, for invaluable comments that have helped to improve and clarify some points.

\section{References}

Aryal, B., \& Saurer, W. 2000, A\&A, 364, L97

Aryal, B., \& Saurer, W. 2004, A\&A, 425, 871

Aryal, B., \& Saurer, W. 2005a, A\&A, 432, 431

Aryal, B., \& Saurer, W. 2005b, A\&A, 432, 841

Aryal, B., \& Saurer, W. 2005c, MNRAS, 360, 125

Djorgovski, S. 1987, in Nearly normal galaxies, ed. S. M. Faber (New York: Springer), 227

Doroshkevich, A. G., \& Shandarin, S. F. 1978, MNRAS, 184, 643

Farouki, R., \& Shapiro, S. L. 1981, ApJ, 243, 32

Flin, P. 1988, MNRAS, 235, 857

Flin, P. 1994, in Cosmological Aspects of X-ray Clusters of Galaxies, ed. W. C. Seitter (Dordrecht: Kluwer), 363

Flin, P. 2001, MNRAS, 325, 49

Flin, P., \& Godlowski, W. 1986, MNRAS, 222, 525

Flin, P., \& Godlowski, W. 1989, Sov. Astron. Lett., 15, 374

Godlowski, W. 1993, MNRAS, 265, 874

Godlowski, W. 1994, MNRAS, 271, 19

Godlowski, W. 1995, Astro. Lett. Commun., 31, 341

Godlowski, W., \& Ostrowski, M. 1999, MNRAS, 303, 50

Godlowski, W., Szydlowski, M., \& Flin, P. 2005, Gen. Relativ. Gravit., 37, 615

Holmberg, E. 1946, Medd. Lund. Astron. Obs, Ser. VI, No. 117

Hu, F. X., Wu, G. X., Su, H. J., \& Liu, Y. Z. 1995, A\&A, 302, 45

Hu, F. X., Yuan, Q. R., Su, H. J., et al. 1998, ApJ, 495, 179

Jaaniste, J., \& Saar, E. 1978, in The Large Scale Structure of the Universe, ed. M. S. Longair, \& J. Einasto (Dordrecht: Reidel), Proc. IAU Symp., 79, 448

Kashikawa, N., \& Okamura, S. 1992, PASJ, 44, 493

Ozernoy, L. M. 1978, in The Large Scale Structure of the Universe, ed. M. S. Longair, \& J. Einasto (Dordrecht: Reidel), Proc. IAU Symp., 79, 427

Peebles, P. J. E. 1969, ApJ, 155, 393

Peebles, P. J. E. 1974, ApJ, 189, L51

Sandage, A., Freeman, K. C., \& Stokes, N. R. 1970, ApJ, 160, 831

Thompson, L. A. 1976, ApJ, 209, 22

Wu, G. X., Hu, F. X., Su, H. J., \& Liu, Y. Z. 1997, A\&A, 323, 317

Yuan, Q. R., Hu, F. X., Su, H. J., \& Huang, K. L. 1997, AJ, 114, 1308

Zeldovich, Ya. B. 1978, in The Large Scale Structure of the Universe, ed. M. S. Longair, \& J. Einasto (Dordrecht: Reidel), Proc. IAU Symp., 79, 409 